\title{
Influência do choro e de padrões respiratórios na deposição de medicação inalatória em crianças
}

\author{
The influence of crying and breathing pattern on inhalatory drug deposition in children
}

Camila Isabel da S. Santos ${ }^{1}$, George Jung da Rosa ${ }^{2}$, Ana Paula Shiratori ${ }^{3}$, Andrezza Brognoli d'Aquino ${ }^{3}$, Geisi Bueno ${ }^{3}$, Renata Tiemi Okuro ${ }^{4}$

RESUMO

Objetivo: Verificar a influência do choro e de padrões respiratórios na eficácia da terapia inalatória em crianças.

Fontes de dados: busca sistemática por artigos científicos referentes ao tema nas bases de dados Cochrane Controlled Trials Data Base, MedLine e Science Direct, publicadas no período de 1994 a 2009. Utilizaram-se os descritores: "choro", "inalação”, "aerossol”, "trabalho respiratório" e "criança", nos idiomas português e inglês.

Síntese dos dados: Foram selecionados 13 artigos, 12 em inglês e um em português. A maioria dos trabalhos apresenta os efeitos da terapia inalatória em crianças, sem discutir a influência do choro e de diferentes padrões respiratórios sobre a deposição da medicação. Estudos que fizeram essa relação verificaram que a respiração e, principalmente, o choro reduzem a quantidade de fármaco que chega às vias aéreas periféricas. Autores discutem as diferenças anatômicas e fisiológicas do sistema respiratório da criança que podem interferir na eficácia da terapia inalatória. Porém, a maioria deles não analisa a influência qualitativa e quantitativa dos padrões respiratórios e do choro sobre a mecânica pulmonar.

Conclusões: $\mathrm{O}$ choro e os padrões respiratórios influenciam na terapia inalatória, sendo atribuída ao choro a redução significativa da deposição medicamentosa nas vias aéreas. Pouco se sabe sobre o princípio determinante para a alteração do potencial de deposição, pois são escassas as evidências sobre o tema, apesar de sua relevância no manejo de afecções pulmonares da população pediátrica.

Palavras-chave: inalação; choro; trabalho respiratório.

\section{ABSTRACT}

Objective: To verify the influence of crying and breathing patterns in the effectiveness of inhalatory therapy in children.

Data sources: Systematic search of scientific studies on the subject in the Cochrane Controlled Trials Database, MedLine and Science Direct, published from 1994 to 2009. The descriptors "crying", "inhalation", "aerosol", "work of breathing" and "child", in Portuguese and in English, were applied.

Data synthesis: 13 studies were selected, 12 in English and one in Portuguese. Most studies reported the effects of inhalation therapy in children, without discussing the influence of crying and breathing patterns on the deposition of the medication. Studies related to this subject found that the respiratory pattern, especially the cry, reduces the amount of drug that reaches the peripheral airways. Authors discuss the anatomical and physiological differences between adults' and children's respiratory system that may interfere with the effectiveness of inhalation. However, most of them do not examine the qualitative and quantitative influence of the breathing patterns and crying on pulmonary mechanics.

Conclusions: Crying and breathing patterns influence the inhalation therapy. The presence of cry significantly reduces the amount of drug deposition in the airways. There is insufficient evidence about the possible mechanisms that explain potential changes of inhalatory drugs deposition in children, despite its relevance to the management of pediatric pulmonary disorders.

Key-words: inhalation; crying; work of breathing.
Instituição: Universidade do Estado de Santa Catarina (UDESC), Florianópolis, SC, Brasil

'Professora Doutora em Saúde da Criança e do Adolescente pela Universidade Estadual de Campinas (Unicamp); Professora Efetiva da Disciplina de Estágio Supervisionado em Pediatria do Curso de Fisioterapia da UDESC, Florianópolis, SC, Brasil

${ }^{2}$ Especialista em Fisioterapia Aplicada à Pediatria pela Unicamp; Professor Colaborador da Disciplina de Estágio Supervisionado em Pediatria do Curso de Fisioterapia da UDESC, Florianópolis, SC, Brasil

${ }^{3}$ Graduanda do Curso de Fisioterapia da UDESC, Florianópolis, SC, Brasil ${ }^{4}$ Mestranda em Saúde da Criança e do Adolescente pela Unicamp; Supervisora dos Cursos de Pós-Graduação em Fisioterapia Aplicada à Pediatria da Unicamp, Campinas, SP, Brasil.
Endereço para correspondência:

Camila Isabel da Silva Santos

Rua Vereador José do Vale Pereira, 37, apto. 301 - Coqueiros CEP 88080-240 - Florianópolis/SC

E-mail: cacaiss@hotmail.com

Conflitos de interesse: nada a declarar

Recebido em: 20/8/2009

Aprovado em: 14/1/2010 


\section{Introdução}

A administração da terapia inalatória (TI) no tratamento de doenças pulmonares tem sido priorizada por permitir a aplicação direta das medicações nas vias aéreas inferiores ${ }^{(1)}$. A TI apresenta resultados clínicos positivos, uma vez que, se administrada apropriadamente, viabiliza que a droga atinja os pulmões e produza a resposta terapêutica espera$\mathrm{da}^{(2)}$. Ao contrário, a medicação consumida por via oral tem grande parte eliminada na primeira etapa do metabolismo, no fígado ${ }^{(3)}$.

Diversos são os aspectos relacionados à efetividade da TI, dentre eles: as propriedades farmacológicas das drogas administradas, as características físicas envolvidas na geração de aerossóis, os aspectos clínicos individuais do paciente, bem como sua cooperação ${ }^{(1,3)}$.

De acordo com Melani ${ }^{(4)}$, existem três tipos de dispositivos geradores de aerossóis: os nebulizadores, os inaladores de pó seco e os inaladores pressurizados dosimetrados. A aerossolterapia no tratamento de desordens respiratórias pediátricas, como asma e fibrose cística, ganhou importância nas últimas décadas e, recentemente, também tem sido introduzida no tratamento de doenças como o diabetes melito, na qual a insulina é aplicada por essa via para diminuir a concentração de glicose no sangue $e^{(5,6)}$.

Os aerossóis são comumente usados em crianças, porém dados sobre a dose correta são escassos e não existe uma abordagem consistente sobre esse cálculo. Algumas drogas são administradas na mesma concentração para crianças de todas as idades, enquanto, para outras, a dose é fundamentada no peso da criança ${ }^{(7)}$. De acordo com Schüepp et a $l^{(3)}$, quando a dosagem é expressa em porcentagem por quilograma de peso corporal, ela independe da idade. Estudos utilizando dispositivos inalatórios têm mostrado que a deposição pulmonar em crianças é relativamente menor do que a do adulto e que está positivamente relacionada ao aumento da idade. A deposição orofaríngea decresce com a idade, principalmente devido a mudanças na anatomia das vias aéreas e no padrão respiratório ${ }^{(3)}$.

A eficácia da TI, isto é, a otimização dos benefícios e a diminuição dos efeitos colaterais sistêmicos, depende da segmentação da droga para as regiões pulmonares relevantes, sendo a deposição pulmonar total o reflexo de tal eficácia. A segmentação é influenciada por características anatômicas e pelo processo patológico instalado nas vias aéreas, além do padrão respiratório e da fórmula da droga utilizada. Fatores psicológicos também interferem na deposição final ${ }^{(3,6)}$.
Apesar de reconhecida a influência do comportamento e de padrões ventilatórios na eficácia da TI, incluindo o choro e a respiração, não há muito conhecimento a respeito da terapia por aerossol em crianças, principalmente em lactentes. Dessa forma, o objetivo deste estudo foi verificar o efeito do choro e dos padrões respiratórios na deposição de medicação inalatória por meio de uma revisão sistemática da literatura.

Realizou-se uma pesquisa de literatura utilizando a base de dados Cochrane Controlled Trials Database, MedLine e Science Direct. As seguintes palavras-chave foram usadas: "choro", "inalação", "aerossol", "trabalho respiratório" e "criança”. Foram selecionados artigos publicados no período de janeiro de 1994 a agosto de 2009, encontrando-se 13 artigos, 12 em inglês e um português. Os critérios de inclusão foram estudos realizados com crianças e compatíveis com o tema proposto.

\section{Anatomia e fisiologia do sistema respiratório da criança}

Os princípios físicos de deposição da medicação utilizada por via inalatória no trato respiratório são semelhantes em lactentes e adultos, sendo a impactação e a sedimentação os mecanismos mais relevantes para a chegada da droga aos pulmões. Uma proporção significante de partículas do medicamento inalado passa pela parte superior e central das vias aéreas e vai se depositar nos pulmões antes de ser exalada, sendo as pequenas partículas as mais propensas a atingir vias aéreas periféricas ${ }^{(8,9)}$.

$\mathrm{Na}$ infância, a distribuição do aerossol é influenciada por características dependentes da idade, como a anatomia das vias aéreas, cujo diâmetro é menor do que no adulto. Esse diâmetro reduzido aumenta a deposição em via aérea central (devido ao alto impacto) e gera menor fluxo inspiratório, o que tende a restringir a possibilidade de impactação ${ }^{(3)}$. Além disso, as vias aéreas dos lactentes são caracterizadas por alta velocidade e fluxo aéreo turbulento, causando aumento da deposição de partículas nas regiões proximais ${ }^{(9)}$. A predominância do padrão nasal para inalação de aerossóis por parte das crianças desse grupo etário também prejudica a deposição. Como o nariz é um filtro muito eficiente, a maior parte do aerossol inalado pelos lactentes e crianças jovens não chega aos pulmões. Apenas as pequenas partículas estão propensas a vencer a passagem nasal e se depositarem em vias aéreas mais distais, quando comparadas às partículas maiores $^{(3,9)}$. 
O aproveitamento da droga inalada é influenciado pelo sistema de deposição dos medicamentos, que está relacionado a fatores como anatomia, fisiologia e tipo de doença pulmonar apresentada pelo paciente. Além disso, a cooperação, a cognição e o desenvolvimento emocional influenciam a quantidade da droga depositada ${ }^{(8)}$. Lactentes só toleram a máscara facial passivamente, devendo-se estar atento quanto ao seu encaixe no rosto, para que não haja escape $^{(3)}$. Crianças com mais idade cooperam ativamente com padrões respiratórios específicos e são capazes de inalar através de um bocal.

Em um estudo realizado por Chua et al ${ }^{(7)}$, verificou-se que aerossóis inspirados por via nasal apresentam deposição pulmonar menor nos lactentes em comparação às crianças mais velhas, o que pode ser decorrente do menor fluxo inspiratório gerado pelos bebês, que inalam somente uma parte da produção nebulizada. As crianças com mais idade inalam quase todo o produto nebulizado.

\section{Choro e padrões respiratórios versus deposição de medicação inalatória}

Lactentes podem não cooperar durante o procedimento de administração de medicação inalatória, ocorrendo choro ou aumento e irregularidade da frequência respiratória. $\mathrm{O}$ choro é parte da fase expiratória e, no lactente, compromete a alternância rítmica da inspiração ${ }^{(10)}$. O choro e o alto fluxo inspiratório diminuem a deposição pulmonar durante a inalação, que fica restrita apenas às partículas menores $^{(9)}$.

No estudo de Nikander $e t$ a $l^{(11)}$, evidencia-se que crianças não gostam e geralmente choram quando são utilizadas máscaras faciais. Com o uso de um modelo específico, foi quantificado o impacto do padrão respiratório choroso na quantidade de droga inalada. A média de volume dependente de todo padrão respiratório foi de $134 \mathrm{~mL}$, com taxa cíclica de 0,26 , sendo que a frequência respiratória não pôde ser definida. Nesse mesmo estudo, verificou-se que o choro reduziu a quantidade de medicação inalada na configuração junto à face, quando comparada aos dados de outras publicações. Em relação à diferença na deposição entre os nebulizadores e o inalador pressurizado dosimetrado, o primeiro sistema tem deposição significativamente menor, situação que os autores relacionam à falta de máscaras faciais vedadas.

Em estudo com 18 crianças, Iles et al ${ }^{(12)}$ observaram que apenas uma fração da medicação inalatória é absorvida pelos pulmões, quando a criança chora. Em concordância, Schüepp et al ${ }^{(3)}$ e Geller ${ }^{(13)}$ verificaram um drástico decréscimo da deposição pulmonar durante o choro e, ainda, um aumento do fluxo inspiratório em cerca de seis a sete vezes. Considerando a influência negativa do choro, Esposito-Festen $e t$ al ${ }^{(14)}$ avaliaram a administração de fármacos inalatórios por meio de aparelho dosimetrado com espaçador, durante o sono de lactentes. No entanto, apesar de essa alternativa proporcionar a colaboração passiva da criança, tema constante de discussão $0^{(10-13)}$, o baixo volume corrente gerado pelo bebê dormindo foi sinônimo de pouco aproveitamento da medicação.

Em síntese, há escassez de estudos recentes com metodologia adequada que avaliem os mecanismos fisiológicos que interferem no potencial de deposição pulmonar por meio da TI. O lactente apresenta diferenças na anatomia e na fisiologia do sistema respiratório em relação ao adulto, as quais desaparecem com o decorrer da idade, mas que interferem na resposta da TI. Algumas hipóteses são sugeridas por especialistas na área para justificar o limitado aproveitamento dessa estratégia terapêutica na população pediátrica, incluindo a maior resistência das vias aéreas na criança, as situações de fluxo aéreo turbulento, como choro e respiração irregular, a respiração nasal ou a falta de entendimento e cooperação. Além desses fatores, é reconhecida a influência química da medicação sobre o mecanismo de clearance da via aérea, principalmente em um sistema ainda em desenvolvimento.

\section{Conclusão}

O choro e os padrões respiratórios da criança influenciam na TI, sendo atribuída ao choro redução significativa na quantidade de deposição medicamentosa em vias aéreas. Ainda são escassas as evidências sobre o tema, apesar de sua relevância no manejo do paciente pediátrico com comprometimento respiratório. 


\section{Referências bibliográficas}

1. Rotta ET, Amantéa SL, Froehlich PE. Principles of the aerosol-therapy in the children acute asthma. Rev AMRIGS 2007;51:70-7.

2. Everard ML. Inhaler devices in infants and children: challenges and solutions. J Aerosol Med 2004;17:186-95.

3. Schüepp KG, Straub D, Möller A, Wildhaber JH. Deposition of aerosols in infants and children. J Aerosol Med 2004;17:153-6.

4. Melani AS. Inhalatory therapy training: a priority challenge for the physician. Acta Biomed 2007;78:233-45.

5. Janssens HM, Tiddens HA. Aerosol therapy: the special needs of young children. Paediatr Respir Rev 2006;7 (Suppl 1):S83-5.

6. Esposito-Festen JE, Zanen P, Tiddens HA, Lammers JW. Pharmacokinetics of inhaled monodisperse beclomethasone as a function of particle size. $\mathrm{Br} \mathrm{J}$ Clin Pharmacol 2007;64:328-34.

7. Chua HL, Collis GG, Newbury AM, Bower GD, Sly PD, Le Souef PN. The influence of age on aerosol deposition in children with cystic fibrosis. Eur Respir J 1994;7:2185-91.
8. Everard ML. Inhalation therapy for infants. Adv Drug Deliv Rev 2003;55:869-78.

9. Janssens HM, De Jongste JC, Hop WC, Tiddens HA. Extra-fine particles improve lung delivery of inhaled sterioids in infants: a study in an upper airway model. Chest 2003;123:2083-8.

10. LaGasse LL, Neal AR, Lester BM. Assessment of infant cry: acoustic cry analysis and parental perception. Ment Retard Dev Disabil Res Rev 2005;11:83-93.

11. Nikander K, Berg E, Smaldone GC. Jet nebulizers versus pressurized metered dose inhalers with valved holding chambers: effects of the facemask on aerosol delivery. J Aerosol Med 2007;20 (Suppl 1):S46-55

12. lles $R$, Lister $P$, Edmunds AT. Crying significantly reduces absorption of aerosolised drug in infants. Arch Dis Child 1999;81:163-5.

13. Geller DE. Comparing clinical features of the nebulizer, metered-dose inhaler, and dry powder inhaler. Respir Care 2005;50:1313-21.

14. Esposito-Festen J, ljsselstijn H, Hop W, van Vliet F, de Jongste J, Tiddens $\mathrm{H}$. Inhaler-Spacer in sleeping young children aerosol therapy by pressured metered-dose. Chest 2006;130:487-92. 